\title{
Pretreatment neutrophil to lymphocyte ratio as prognostic factor in metastatic breast cancer treated with cyclin dependent kinase 4/6 inhibitors
}

Pauline Rottier ( $\sim$ pau.rottier@gmail.com )

Centre Francois Baclesse Centre de Lutte Contre le Cancer https://orcid.org/0000-0002-2420-3350

Angélique DA SILVA

Centre Francois Baclesse Centre de Lutte Contre le Cancer

Alison JOHNSON

Centre Francois Baclesse Centre de Lutte Contre le Cancer

Christelle LEVY

Centre Francois Baclesse Centre de Lutte Contre le Cancer

Djelila ALLOUACHE

Centre Francois Baclesse Centre de Lutte Contre le Cancer

loana HRAB

Centre Francois Baclesse Centre de Lutte Contre le Cancer

\section{Carine SEGURA}

Centre Francois Baclesse Centre de Lutte Contre le Cancer

Adeline MOREL

Centre Francois Baclesse Centre de Lutte Contre le Cancer

Maud VILLEMIN

Centre Francois Baclesse Centre de Lutte Contre le Cancer

Clémence BOSCHER

Centre Francois Baclesse Centre de Lutte Contre le Cancer

Marion BERTHO

Centre Francois Baclesse Centre de Lutte Contre le Cancer

Louis BOISMOREAU

Centre Francois Baclesse Centre de Lutte Contre le Cancer

François CHERIFI

Centre Francois Baclesse Centre de Lutte Contre le Cancer

Justine LEQUESNE

Centre Francois Baclesse Centre de Lutte Contre le Cancer

George EMILE

Centre Francois Baclesse Centre de Lutte Contre le Cancer 


\section{Research Article}

Keywords: Metastatic breast cancer, NLR. cyclin dependent kinase inhibitor. prognostic factor

Posted Date: February 4th, 2022

DOI: https://doi.org/10.21203/rs.3.rs-1315803/v1

License: (c) (i) This work is licensed under a Creative Commons Attribution 4.0 International License. Read Full License 


\section{Abstract}

Background: Cyclin dependent kinase inhibitors (CdK4/6i) changed the course of hormone receptor positive (HR+) HER2negative (HER2-) metastatic breast cancer (mBC). To date, no factors have been shown to predict response to CdK4/6i. Neutrophil-to-lymphocyte ratio (NLR), an indicator of the host systemic inflammatory response, is an independent prognostic factor for survival in cancers. We conducted this study to evaluate the addition of NLR for survival assessment in $\mathrm{mBC}$ patients treated with first line CdK4/6i.

Methods: All mBC patients treated with first line CdK4/6i between November 2015 and December 2019 were retrospectively included. We analyzed 12-month progression free survival (PFS), overall survival (OS) and response rate according to NLR in univariable and multivariable analysis.

Results: A total of 126 patients treated with palbociclib $(n=101)$, ribociclib $(n=18)$ or abemaciclib $(n=7)$ were included, with a median follow-up of 16 months [range: 2.9-37]. Median age was 65 years [29-86], $88 \%$ patients had good performance status (0-1). Most patients were included at the metastatic relapse stage $(71 \%)$ and $20 \%$ had visceral metastases without bone lesions. High NLR ( $\geq 2.53)$ was significantly associated with worse PFS (Hazard Ratio $\left.(\mathrm{HR})=0.52, \mathrm{Cl}_{95 \%}=[0.29-0.94]\right)$. Fourteen patients died (11.1\%). Complete responses were more frequent in the low NLR group (<2.53) $(14.5 \%$ vs. $3.1 \%, p=0.029)$.

Conclusion: High NLR is associated with worse PFS in HR+ HER2- mBC patients treated with first line CdK4/6i. NLR is a reliable and inexpensive prognostic marker, easily accessible in routine clinical practice, which could optimize the therapeutic strategy. These results need to be confirmed in larger prospective studies.

\section{Introduction}

Breast cancer (BC) is the most common malignancy among women and one of the leading causes of death by cancer worldwide [1] despites effective early detection methods and new therapeutic advances. Around $6-10 \%$ of $B C$ are diagnosed with de novo metastatic disease and $25-30 \%$ present a metastatic relapse [2]. Metastatic breast cancer $(\mathrm{mBC})$ has a poor survival with a 5-year relative survival rate dropping to around $38 \%$ vs. $96 \%$ for early BC (eBC), in Europe [3]. Approximately $70 \%$ of BC are hormone receptor positive $(\mathrm{HR}+)$ and human epidermal growth factor receptor 2 negative (HER2-). Endocrine therapy (ET) is the main treatment for patients with HR+/HER2- mBC. The advent of cyclin-dependent kinase inhibitors (CdK4/6i) has considerably improved the prognosis and they are now the gold standard for first line treatment of HR+/HER2- mBC without extensive visceral involvement [3-6].

Prognostic factors are important in estimating outcomes and identifying the optimal treatment for each patient. Some clinical or histological markers are commonly used and validated in HR+/HER2- mBC such as poor Eastern Cooperative Oncology Group Performance Status (ECOG-PS), higher tumor grade, Ki67 level, negative progesterone receptor (PR) status, prior therapy, sites and number of metastases (multiple vs single), and shorter time to progression to $\mathrm{mBC}$ [7]. The choice of first-line treatment is crucial, as it 
affects patients' outcome. However, no predictive factor of response to CdK4/6i and ET has been identified. Biomarkers are needed to help personalize first line treatment.

Over the last decade, host systemic inflammatory response have been shown to be involved in tumor growth, invasion, angiogenesis and progression $[8,9]$. This inflammation could be assessed by pretreatment peripheral differential leukocyte count and the calculation of more informative ratios such as neutrophil-to-lymphocyte ratio (NLR), platelet-to-lymphocyte ratio (PLR), lymphocyte-to-monocyte ratio (LMR) and estimation of lymphocyte count. Several studies in different stages of solid cancers [10-12], including $\mathrm{BC}$, evaluated these ratios and they are now acknowledged as predictive and prognostic factors. In a metanalysis, it was highlighted that high pretreatment NLR was an independent poor prognostic factor for overall survival (OS) and progression-free survival (PFS) in all-stage BC, with a strongest association in the HR+/HER2- subgroup [13]. Koh et al. [14] revealed in a prospective study that both NLR and PLR are independently associated with an increased risk of mortality in all-stage BC. However, these inflammation biomarkers have mostly been evaluated in the (neo)adjuvant chemotherapy setting for eBC $[15,16]$, particularly in triple-negative BC (TNBC) [17]. Data remains limited and inconsistent for $\mathrm{mBC}$ [18]. Concerning LMR, high LMR before neoadjuvant chemotherapy was reported as a favorable prognostic factor in eBC regardless of HR/HER2 status [19], but no data has been reported for $\mathrm{mBC}$ HR+/HER2-.

The aim of our study was to assess the prognostic impact of peripheral leukocyte count, NLR, lymphopenia, PLR and LMR on survival and response rates in women receiving first line CdK4/6i in association with ET for locally advanced or $\mathrm{mBC}$.

\section{Methods}

\section{Population}

We carried out a retrospective single center study at the Comprehensive Cancer Center François Baclesse in Caen, France, as recommended by REMARK for the evaluation of prognostic tumor marker [20]. All adult women who received CdK4/6i for histologically proven HR+/HER2- locally advanced or mBC from November 2015 to December 2019 were included. Patients receiving any of the European Medicines Agency (EMA) or Food and Drug Administration (FDA)-approved CdK4/6i (palbociclib, ribociclib, abemaciclib) in association with ET as first-line treatment were included. Patients were excluded if they had received prior first-line treatment or presented with visceral crisis.

\section{Endpoints}

We collected the general characteristics of patients (e.g., age, ECOG-PS, menopausal status) and their disease (e.g., TNM staging, hormone receptor expression and SBR (Scarff Bloom Richardson) grade from the primary tumor site or a current metastatic lesion). Results of the blood test performed 24 to 48 hours before starting treatment were collected. NLR, PLR and LMR calculation was performed. NLR was defined as the absolute neutrophil count divided by the absolute lymphocyte count, PLR was defined as the 
absolute platelet count divided by the absolute lymphocyte count and LMR was defined as the absolute lymphocyte count divided by the absolute monocyte count. Lymphopenia was defined by lymphocyte count below 1.5G/L. Tumor imaging (by computed tomography scan) was performed every 2-3 cycles and disease response was classified by the radiologist according to the Response Evaluation Criteria in Solid Tumors (RECIST, version 1.1 [21]) as complete response (CR), partial response (PR), stable disease (SD) or progressive disease (PD). Objective response rate (ORR) corresponded to the proportion of patients in whom a CR or PR was observed. Disease control rate (DCR) represented the percentage of patients with either $\mathrm{CR}, \mathrm{PR}$, or SD as the best overall response. The Progression-Free Survival was defined as the time elapsed between CdK4/6i initiation and radiological progression, death or lost to follow-up, Adverse events (AE) collected at each medical visit were graded according to National Cancer Institute Common Terminology Criteria for Adverse Events (NCI CTCAE) version 5.0.

\section{Objectives}

The primary objective was to assess the 12-month PFS rate according to pretreatment NLR, PLR, LMR and lymphopenia. Secondary objectives included assessment of Overall Survival (OS), ORR and DCR, according to NLR, PLR, LMR and lymphopenia, and safety.

Statistical analysis: Descriptive analysis of data was provided, for qualitative variables as frequencies; for quantitative variables as median and extreme values. Survival curves were estimated by the Kaplan Meier method, and compared by the log-rank test. Multivariable analysis for PFS and OS was performed using Cox's proportional hazards regression model including biological markers significantly associated with survival at a significance level of 0.10 and adjusted on clinical parameters. A stepwise model selection was performed through Akaike's Information Criterion optimization, corresponding to significance-based selection at a significance level of 0.157 . The optimal cut-off values for the NLR, PLR and LMR to predict 1-year progression were determined by maximizing the product of sensitivity and specificity, through receiver operating characteristics (ROC) curve analysis. The characteristics of high NLR and low NLR patients were compared by $\chi 2$ test (or Fisher's exact test, in case of observed values per category $<5$ ) for the qualitative variables, and by the Student's t-test for the quantitative variables (or Wilcoxon non-parametric test if data were not normally distributed). Statistical tests and confidence intervals were calculated with an overall risk of first kind error of $5 \%$. All incident cases were assessed (no calculation of the number of subjects needed). Analyses were conducted using R software, version 4.0.2 (https://cran.r-project.org/bin/windows/base/).

\section{Ethic}

The study was in accordance with national regulations regarding research involving human subjects. Registration in the CIL (correspondant informatique et libertés) register was carried out for this study. Patients non-opposition to the use of their data was sought after verification of vital status. All data were anonymized for statistical analysis. 


\section{Results}

Population: From November 2015 to December 2019, 126 patients were included, with a median followup of 16 months (range, 2.9 to 37) (Fig. 1: Flow-chart). The median age at inclusion was 65 years (range, 29 to 86$)$. At diagnosis of $\mathrm{mBC}, 101$ patients (80.1\%) had bone metastases and 86 (68.3\%) had visceral disease. Thirty-five (29.2\%) patients presented with de novo $\mathrm{mBC}$ and $31 \%$ had not received any previous treatment except for surgery. The most commonly prescribed CdK4/6i was palbociclib ( $n=101,80 \%)$, followed by ribociclib $(n=18,14 \%)$ and abemaciclib $(n=7,6 \%)$, combined with ET (aromatase inhibitor $+/$ LHRH analogue for 104 patients (82.5\%) or fulvestrant for 22 patients $(17.5 \%)$ ). Patient characteristics are presented in Table 1. 
Table 1

Patient characteristics at baseline

\begin{tabular}{|c|c|}
\hline Population characteristics & $N=126(\%)$ \\
\hline Median age, years [range] & $65[29 ; 86]$ \\
\hline ECOG PS & $50(39.7)$ \\
\hline 0 & $62(49.2)$ \\
\hline 1 & $12(9.5)$ \\
\hline 2 & $2(1.6)$ \\
\hline \multicolumn{2}{|l|}{3} \\
\hline Histology at diagnosis & $94(74.6)$ \\
\hline Ductal & $29(23.0)$ \\
\hline Lobular & $3(2.4)$ \\
\hline \multicolumn{2}{|l|}{ Others } \\
\hline SBR grade at diagnosis & $18(14.3)$ \\
\hline I & $72(57.1)$ \\
\hline II & $32(25.4)$ \\
\hline III & $4(3.0)$ \\
\hline \multicolumn{2}{|l|}{ Unknow } \\
\hline Stage at diagnosis & $13(10.8)$ \\
\hline I & $37(30.8)$ \\
\hline II & $34(27)$ \\
\hline III & $36(28.6)$ \\
\hline IV & $6(4.8)$ \\
\hline \multicolumn{2}{|l|}{ Unknown } \\
\hline Menopause & $94(74.6)$ \\
\hline Yes & $32(25.4)$ \\
\hline \multicolumn{2}{|l|}{ No } \\
\hline De novo metastatic cancer & $36(28.6)$ \\
\hline Yes & $90(71.4)$ \\
\hline No & \\
\hline
\end{tabular}




\begin{tabular}{|c|c|}
\hline Population characteristics & $N=126(\%)$ \\
\hline Metastatic sites & $3(2.4)$ \\
\hline Locoregional & $101(80.1)$ \\
\hline Bone & $86(68.3)$ \\
\hline \multicolumn{2}{|l|}{ Visceral } \\
\hline Prior therapy ${ }^{a}$ & $87(69)$ \\
\hline Yes & $39(31)$ \\
\hline \multicolumn{2}{|l|}{ No } \\
\hline NLR (cut-off = 2.53) & $64(51)$ \\
\hline High & $62(49)$ \\
\hline \multicolumn{2}{|l|}{ Low } \\
\hline Lymphopenia (< 1.5G/L) & $59(47)$ \\
\hline Yes & $67(53)$ \\
\hline \multicolumn{2}{|l|}{ No } \\
\hline PLR (cut-off = 174.4) & $68(54)$ \\
\hline High & $58(46)$ \\
\hline \multicolumn{2}{|l|}{ Low } \\
\hline LMR (cut-off = 3.3) & $60(48)$ \\
\hline High & $66(52)$ \\
\hline \multicolumn{2}{|l|}{ Low } \\
\hline \multicolumn{2}{|c|}{$\begin{array}{l}\text { ECOG-PS = Eastern Cooperative Oncology Group }- \text { Performance Status; } S B R=\text { Scarff-Bloom- } \\
\text { Richardson; NLR = Neutrophil to Lymphocyte Ratio; } P L R=\text { Platelet to } L y m p h o c y t e \text { Ratio; } L M R= \\
\text { Lymphocyte to Monocyte Ratio. }\end{array}$} \\
\hline
\end{tabular}

\section{Overall population outcomes}

The 12-month PFS rate was $72.6 \%\left(\mathrm{Cl}_{95 \%}=\right.$ [64.8-81.3]), with a median PFS time of 27 months. Fourteen patients were dead at the end of the follow-up. The 12-month OS rate was $94.7 \%\left(\mathrm{Cl}_{95 \%}=\right.$ [90.6-98.9]), and median OS was not reached. At 12 months, DCR was 92\% (11 RC, 63 PR and 42 SD, i.e. 116 patients) and ORR was $58.7 \%$ (74 patients). 
Prognostic value of NLR: The optimal NLR cut-off value to predict progression within 12 months after metastatic diagnosis was 2.53; 64 patients (50.8\%) were classified in the high NLR group (NLR $\geq 2.53$ ). The rate of pretreatment ECOG-PS 0 patients was higher in the low NLR group than in the high NLR group $(51.6 \%$ vs. $28.1 \%, p=0.012)$. No other difference in patient characteristics was observed according to NLR (Table 2). The 12-month PFS rate for the low NLR group was 78.6\% [ $\left.\mathrm{Cl}_{95 \%}: 68.4-90.2\right]$ versus $66.8 \%$ [55.8$80.0]$ for the high NLR group (log-rank $p=0.027$ ) (Table 3 ). PFS was significantly worse in the high NLR group with a median of 17 months, while it was not achieved in the low NLR group ( $\mathrm{HR}=0.52,\left[\mathrm{Cl}_{95 \%}\right.$ : 0.29 0.94]) (Fig. 2: PFS probability according to pretreatment NLR). No difference between the two groups was observed for OS.

Table 2

Patient characteristics at baseline according to NLR groups

\begin{tabular}{|lllll|}
\hline Variable & N & NLR & p value \\
\hline & & High $(\geq 2.53)$ & Low $(<2.53)$ & \\
& & $\mathrm{n}=64$ & $\mathrm{n}=62$ & \\
\hline Median age, years [range] & 126 & $65[29 ; 86]$ & $65.5[32 ; 83]$ & 0.58 \\
\hline ECOG PS & 50 & $18(28.1 \%)$ & $32(51.6 \%)$ & 0.012 \\
0 & 76 & $46(71.9 \%)$ & $30(48.4 \%)$ & \\
1,2 or 3 & & & & 0.47 \\
\hline Postmenopausal patients & 94 & $50(78.1 \%)$ & $44(71 \%)$ & 0.39 \\
\hline De novo stage IV disease & 36 & $15(25 \%)$ & $21(33.9 \%)$ & 0.15 \\
\hline Bone metastases & 101 & $55(85.9 \%)$ & $46(74.2 \%)$ & 0.69 \\
\hline Prior therapy for eBC ${ }^{\text {a }}$ & 31 & $18(28.1 \%)$ & $21(33.9 \%)$ & \\
\hline eBC = early Breast Cancer & & & & \\
\hline a including chemotherapy and/or radiotherapy and/or endocrine therapy. & \\
\hline
\end{tabular}


Table 3

12-month PFS rate according to biomarkers

\begin{tabular}{|c|c|c|c|c|}
\hline Variable & $\mathbf{N}$ & Number at risk (12 months) & 12-month PFS rate & $\mathrm{Cl}_{95 \%}$ \\
\hline \multicolumn{5}{|l|}{ NLR } \\
\hline$\geq 2.53$ & 64 & 33 & 66.8 & {$[55.8-80.0]$} \\
\hline$<2.53$ & 62 & 37 & 78.6 & {$[68.4-90.2]$} \\
\hline \multicolumn{5}{|l|}{ Lymphopenia } \\
\hline$<1.5 \mathrm{G} / \mathrm{L}$ & 59 & 33 & 68.9 & {$[58.1-81.8]$} \\
\hline$\geq 1.5 \mathrm{G} / \mathrm{L}$ & 67 & 37 & 76.6 & {$[66.2-88.7]$} \\
\hline \multicolumn{5}{|l|}{ PLR } \\
\hline$\geq 174.4$ & 68 & 38 & 69.5 & {$[58.7-82.1]$} \\
\hline$<174.4$ & 58 & 32 & 76.6 & {$[66.2-88.7]$} \\
\hline \multicolumn{5}{|l|}{ LMR } \\
\hline$\geq 3.3$ & 60 & 31 & 68.7 & {$[57.2-82.5]$} \\
\hline$<3.3$ & 66 & 39 & 76.1 & {$[66.1-87.5]$} \\
\hline
\end{tabular}

Distribution of response was significantly different between the low NLR group and the high NLR group $(p=0.026)$, with better response in the low NLR (Table 4). ORR was $59.7 \%$ in the low NLR group and $57.8 \%$ in the high NLR group; DCR was $96.8 \%$ and $85.9 \%$ respectively. Especially, there were more CR in the low NLR group $(n=9,14.5 \%)$ than in the high NLR group $(n=2,3.1 \%)$. 
Table 4

Best response according to NLR and lymphopenia

\begin{tabular}{|lcccccr|}
\hline Variable & N & CR (\%) & PR (\%) & SD (\%) & PD (\%) & p value \\
\hline NLR & & & & & & $0.026^{\mathrm{a}}$ \\
\hline$\geq 2.53$ & 64 & $2(3.1)$ & $35(54.7)$ & $19(29.7)$ & $8(12.5)$ & \\
\hline$<2.53$ & 62 & $9(14.5)$ & $28(45.2)$ & $23(37.1)$ & $2(3.2)$ & \\
\hline Lymphopenia & & & & & & 0.32 \\
\hline$<1.5$ G/L & 67 & $3(4.5)$ & $36(53.7)$ & $22(32.8)$ & $6(9.0)$ & \\
\hline$\geq 1.5$ G/L & 59 & $8(13.6)$ & $27(45.8)$ & $20(33.9)$ & $4(6.8)$ & \\
\hline $\begin{array}{l}\text { NLR }=\text { Neutrophil to Lymphocyte Ratio; } \\
\text { a } p<0.05\end{array}$ & & & & & \\
\hline
\end{tabular}

Prognostic value of lymphopenia: Sixty-seven patients (53\%) had baseline lymphopenia. Lymphopenia was significantly associated with shorter PFS of 19 months versus 31 months for patients with normal absolute lymphocyte count (ALC) (log-rank $p=0.041$ ) (Fig. 3: PFS probability according to pretreatment ALC). The 12-month PFS rate was better in the normal ALC group with $76.6 \%\left[\mathrm{Cl}_{95 \%}: 66.2-88.7\right]$ versus $68.9 \%\left[\mathrm{Cl}_{95 \%}: 58.1-81.8\right]$ for patients with lymphopenia (Table 3). OS, ORR and DCR were not associated with baseline lymphopenia.

Prognostic value of other ratios: The determined PLR cut-off was 174.4 with 68 patients $(54 \%)$ in the high PLR group. Pretreatment PLR did not influence PFS ( $\mathrm{HR}=0.80,\left[\mathrm{Cl}_{95 \%}: 0.451-1.426\right]$ with median PFS of 27 months and 20 months in the low and high PLR groups, respectively) and OS. The 12-month PFS rate was greater in the low PLR group with $76.6 \%$ vs. $69.4 \%$ in the high PLR group but this difference was not statistically significant.

The LMR cut-off was 3.3. Pretreatment LMR did not influence 12-month PFS rate (Table 3) or OS.

As the results of PLR and LMR were not significant on PFS, the analysis of the response rate was not performed.

\section{Multivariable analysis}

In multivariable analysis, NLR< 2.53 and lymphopenia were included in the model, with adjustment on dose reduction, occurrence of grade $3 / 4$ toxicity, de novo metastatic cancer, bone metastases, SBR grade, RP status and ECOG-PS status. Selection model retained NLR<2.53 and ECOG PS as independently associated with PFS, with respectively $p=0.087$ and $p=0.068$. Especially, NLR $<2.53$ was associated with a $\mathrm{HR}=0.59$ ([0.32-1.08]) and an impaired of general status (ECOG-PS 1, 2 or 3 ) was associated with $\mathrm{HR}=1,79$ [0.96-3.36] in multivariable Cox model (supplementary Table 1). 


\section{Safety}

The most frequently reported AE were hematologic toxicities with anemia ( $n=79$ patients, $62.7 \%)$, neutropenia $(n=110,87.3 \%)$ and thrombocytopenia $(n=38,30.2 \%)$. Grade $3 / 4$ neutropenia was observed in 57 patients (45\%). Only few patients experienced grade $3 / 4$ anemia $(n=12,9.5 \%)$ and thrombocytopenia $(n=2,1.6 \%)$. Dose reductions were required for 52 patients $(41.3 \%)$.

\section{Discussion}

In this retrospective study, we highlighted that pretreatment high NLR $(\geq 2.53)$ and lymphopenia (ALC< $1.5 \mathrm{G} / \mathrm{L}$ ) were prognostic markers associated with poorer PFS in women treated with first-line CdK4/6i and ET for metastatic or locally advanced HR+/HER2- breast cancer.

No consensus has been reached to define a cut-off or threshold value for each ratio (NLR, PLR, LMR). We first determined these cut-offs with ROC curves. In our study, NLR cut-off was similar to those found in the literature mostly ranging between 2 and 5 . A meta-analysis conducted in BC reported a median NLR cut-off value of 2.5 in 10 out of 15 studies [13].

Our results are consistent with previous studies. Four recent meta-analyses corroborate our findings showing that NLR is an independent prognostic factor for PFS and OS in patients with BC at different stages [13, 22, 23], especially for luminal A subtype [24]. Wariss et al. [25] reported an association between high NLR and worse OS in 2,374 eBC and $\mathrm{mBC}$ patients, but only for patients with luminal subtypes. In another study concerning mTNBC, NLR> 2.5 at diagnosis was a useful predictor of poor OS, regardless of the subsequent treatment [26]. In HR+/HER2- eBC, high NLR (>2.25) after neoadjuvant chemotherapy was correlated with poorer disease free survival (DFS) and OS, especially in patients with non-pathologic complete response (pCR) [15]. Furthermore, NLR has been evaluated as a prognostic factor for other endpoints besides survival in different tumor types. For example, it was shown in esophageal cancer that patients with high NLR $(\geq 2.2)$ had lower pCR rates (and a delayed response to chemotherapy) compared to those with a NLR< $2.2(21 \%$ vs. $56 \%, p=0.001)$ [27]. To date, our study is the first to report the prognostic impact of NLR on survival and response rates to first line treatment for HR+/HER2- $m B C$.

Low NLR appears to be a protective factor for progression-free survival rate with a $40 \%$ risk reduction of progression or death ( $\mathrm{HR}=0.59$ [ $\left.\left.\mathrm{Cl}_{95 \%}: 0.32-1.08\right]\right)$, independently of ECOG-PS status in multivariable analysis. However, this result was no significant probably due to the lack of power resulting from a limited number of events as well as PFS results were not mature enough to support these. Similarly, we could not validate ECOG-PS as a significant independent predictive factor of disease progression, although this has been acknowledged in the literature.

Lymphopenia and NLR are two complementary prognostic factors. Increased systemic inflammation markers have been reported in lymphopenic patients, with an inverse increase in the percentage of 
peripheral neutrophils, due to the expression of pro-inflammatory cytokines such as IL-6 and IL-7, CD4+CD8+ double-positive (DP) thymocytes and age-related thymic function [28].

Lymphopenia is multifactorial and can be associated with patient characteristics (as age, ECOG-PS) [28] or tumor burden. In our study, the median age was similar between the two groups of pretreatments NLR, but they differed on ECOG-PS: patients in the high NLR group had a worse ECOG-PS, that was associated with a lower PFS. Otherwise, cell death secondary to inflammation and reduced thymic function have been suggested as possible mechanisms of peripheral lymphopenia observed in metastatic patients [29].

Lymphocytes, whether in peripheral blood or as tumor-infiltrating lymphocytes, play a major role in controlling disease progression. For example, in patients receiving everolimus for $\mathrm{mBC}$, Schettini et al. reported that responders had a higher number of circulating CD8+ and CD4+ T cells, and increased pretreatment infiltration of these cells in the tumor microenvironment than non-responders [30]. Concerning patients treated with palbociclib for HR+/HER2- mBC, we previously showed that those with pretherapeutic ALC $<1.5 \mathrm{G} / \mathrm{L}$ had significantly shorter PFS time ( 6 vs. 10 months, $p=0.004$ ), shorter OS time (20 vs. 33 months, $p=0.018)$ and more disease progression at first imaging evaluation [31].

It is necessary to thoroughly understand the impact of the immune system on tumor control. On the one hand, neutrophils, B lymphocytes and some CD4+ T cells may stimulate cancer growth. On the other hand, cytotoxic CD8+ T cells are crucial components of tumor-specific cellular adaptive immunity as Thelper 1 (TH1), TH17, CD4+ T cells and Natural Killer cells are in the tumor microenvironment are. They inhibit tumor growth by producing interferon gamma, subsequently leading to angiostasis, cell cycle inhibition, apoptosis and tumor phagocytosis by macrophages [9]. A retrospective study of 1,902 patients with $\mathrm{eBC}$ showed that a high total and peripheral CD8+ T cell count was associated with significantly longer breast cancer-specific survival (BCSS) [32]. More specifically, in patients with ER-positive tumor, the total number of infiltrating CD8+ T cells was not significantly associated with patient outcome, whereas peripheral CD8+ count was associated with longer BCSS [32]. Furthermore, Coffelt et al. demonstrated that elevated neutrophil counts induced by BC tumor cells suppressed CD8+ T cells and promoted metastasis through immunosuppression [33].

Furthermore, CdK4/6i have been reported to increase tumor immunogenicity by overcoming two principal mechanisms of tumor immune evasion. They limit the proliferation of Regulatory $T$ cells (Tregs) leading to reduced immunosuppression and enhance antitumor immunity by increasing $T$ cell activation, promoting $T$ cell tumor infiltration, and expanding the functional capacity of tumor cells to present antigens [34, 35].

Other biomarkers evaluated in our study, such as LMR and PLR, had no impact on PFS or OS. Nevertheless, after 12 months of treatment, patients with high PLR ( $\geq 174)$ had a non-significant shorter median PFS than patients with low PLR. PLR has been described as a reliable prognostic marker in many cancers including BC [36]. One study identified low pretreatment PLR value to be an independent predictive factor of pCR and DFS in patients with luminal B-like (HER2-) BC, suggesting that patients with a low platelet count and high lymphocyte count exhibit increased antitumor activity [37]. In luminal BC, 
PLR> 185.5 had a strong prognostic value for DFS in patients with lymph node metastases [38]. Platelets promote angiogenesis and invasiveness of tumor cells by enhanced metalloproteinase-9 (MMP-9) secretion, degradation of the extracellular matrix, release of adhesion molecules and growth factors such as VEGF-A or PDGF $[39,40]$. Also, IL-6 implicated in the systemic inflammatory response and recruitment of neutrophils, can stimulate differentiation and multiplication of megakaryocytes [41]. Concerning LMR, it was associated with poor OS in a meta-analysis of patients with solid tumors [42]. In the BC neoadjuvant chemotherapy setting, a recent study confirmed this result in a multivariable analysis and showed that patients with low LMR had shorter DFS [43].

None of the parameters we studied had an impact on OS possibly due to limited follow-up with an OS not mature. Of note, the median PFS of 27 months in this study was similar to that obtained in the registration trials of CdK4/6 inhibitors [5].

This study had some limitations. Due to the retrospective nature of the study, we were unable to collect the values of certain inflammation parameters (albumin, C-reactive protein, vitamin D, and LDH) or data concerning concomitant treatments (e.g., corticosteroid therapy) and radiotherapy, which may influence neutrophil and lymphocyte counts $[44,45]$. The sample size is limited and results must be interpreted with caution. Moreover, PLR and LMR were not significant on the primary outcome possibly due to the small sample size and a lack of power but also because the cut-off determined was not sufficiently discriminating. Is that it prompted us to design a prospective study, in order to improve and to confirm our results more powerfully.

Immune status is emerging as an essential biomarker of the tumor biology and microenvironment with an impact on patient outcome. Other biomarkers, such as tumor infiltrating lymphocytes (TILS) and circulating tumor cells (CTC), are still being evaluated in clinical research as prognostic factors but are not easily obtained in routine clinical practice [7].

Our study shows that NLR and ALC, indicators of the immune status, are interesting biomarkers in routine clinical practice because they are both available, easy-to-use, reliable and inexpensive prognostic factors of PFS in mBC patients treated with CdK4/6i in the first-line setting. These results could eventually help select patients more likely to benefit from CdK4/6i. These findings need to be confirmed in a larger prospective study.

\section{Abbreviations}

$\mathrm{BC}$

Breast Cancer

$\mathrm{HR}+$

Hormone Receptor positive

HER2-

Human Epidermal growth factor Receptor 2 negative

TNBC 
Triple-Negative Breast Cancer

ET

Endocrine Therapy

NLR

Neutrophil-to-Lymphocyte Ratio

PLR

Platelet-to-Lymphocyte Ratio

LMR

Lymphocyte-to-Monocyte Ratio

EMA

European Medicines Agency

FDA

Food and Drug Administration

ECOG PS

Eastern Cooperative Oncology Group - Performance Status

HR

Hazard ratio

\section{Declarations}

\section{Funding:}

The authors declare that no funds, grants, or other support were received during the preparation of this manuscript.

\section{Competing Interests:}

The authors have no relevant financial or non-financial interests to disclose. None to declare in relation to the presented study.

\section{Author Contributions:}

EMILE George, DA SILVA Angélique and ROTTIER Pauline contributed to the study conception and design. Material preparation, data collection and analysis were performed by ROTTIER Pauline. LEQUESNE Justine conducted the statistics. The first draft of the manuscript was written by ROTTIER Pauline and DA SILVA Angélique, and all authors commented on previous versions of the manuscript. All authors read and approved the final manuscript.

\section{Data Availability:}

The datasets generated during and/or analyzed during the current study are not publicly available due to the medically confidential nature of the data but are available from the corresponding author on reasonable request. 


\section{Ethics approval and Consent to participate:}

This is an observational study. The local Research Ethics Committee has confirmed that no ethical approval is required. In accordance with the regulations regarding research involving human subjects, the present study was registered with corresponding data protection. Patients' non-opposition to the use of their data was sought after verification of their vital status.

\section{References}

1. The global Cancer, Observatory (2019) May

2. Dawood S, Broglio K, Ensor J et al (2010) Survival differences among women with de novo stage IV and relapsed breast cancer. Ann Oncol 21:2169-2174. https://doi.org/10.1093/annonc/mdq220

3. Gennari A, André F, Barrios CH et al (2021) ESMO Clinical Practice Guideline for the diagnosis, staging and treatment of patients with metastatic breast cancer. https://doi.org/10.1016/j.annonc.2021.09.019. Ann Oncol S0923753421044987

4. Cardoso F, Senkus E, Costa A et al (2018) 4th ESO-ESMO International Consensus Guidelines for Advanced Breast Cancer (ABC 4). Ann Oncol 29:1634-1657. https://doi.org/10.1093/annonc/mdy192

5. Finn RS, Martin M, Rugo HS et al (2016) Palbociclib and Letrozole in Advanced Breast Cancer. N Engl J Med 375:1925-1936. https://doi.org/10.1056/NEJMoa1607303

6. Cardoso F, Kyriakides S, Ohno S et al (2019) Early breast cancer: ESMO Clinical Practice Guidelines for diagnosis, treatment and follow-up. Ann Oncol 30:1194-1220. https://doi.org/10.1093/annonc/mdz173

7. Cuyún Carter G, Mohanty M, Stenger K et al (2021) Prognostic Factors in Hormone ReceptorPositive/Human Epidermal Growth Factor Receptor 2-Negative (HR+/HER2-) Advanced Breast Cancer: A Systematic Literature Review. Cancer Manag Res Volume 13:6537-6566. https://doi.org/10.2147/CMAR.S300869

8. Coussens LM, Werb Z (2002) Inflammation and cancer. Nature 420:860-867. https://doi.org/10.1038/nature01322

9. Pagès F, Galon J, Dieu-Nosjean M-C et al (2010) Immune infiltration in human tumors: a prognostic factor that should not be ignored. Oncogene 29:1093-1102. https://doi.org/10.1038/onc.2009.416

10. Guthrie GJK, Charles KA, Roxburgh CSD et al (2013) The systemic inflammation-based neutrophillymphocyte ratio: Experience in patients with cancer. Crit Rev Oncol Hematol 88:218-230. https://doi.org/10.1016/j.critrevonc.2013.03.010

11. Clarke SJ, Chua W, Moore M et al (2011) Use of Inflammatory Markers to Guide Cancer Treatment. Clin Pharmacol Ther 90:475-478. https://doi.org/10.1038/clpt.2011.122

12. Templeton AJ, McNamara MG, Šeruga B et al (2014) Prognostic Role of Neutrophil-to-Lymphocyte Ratio in Solid Tumors: A Systematic Review and Meta-Analysis. JNCI J Natl Cancer Inst 106. https://doi.org/10.1093/jnci/dju124 
13. Ethier J-L, Desautels D, Templeton A et al (2017) Prognostic role of neutrophil-to-lymphocyte ratio in breast cancer: a systematic review and meta-analysis. Breast Cancer Res 19:2. https://doi.org/10.1186/s13058-016-0794-1

14. Koh C-H, Bhoo-Pathy N, Ng K-L et al (2015) Utility of pre-treatment neutrophil-lymphocyte ratio and platelet-lymphocyte ratio as prognostic factors in breast cancer. Br J Cancer 113:150-158. https://doi.org/10.1038/bjc.2015.183

15. Koh YW, Lee HJ, Ahn J-H et al (2014) Prognostic significance of the ratio of absolute neutrophil to lymphocyte counts for breast cancer patients with ER/PR-positivity and HER2-negativity in neoadjuvant setting. Tumor Biol 35:9823-9830. https://doi.org/10.1007/s13277-014-2282-5

16. Ma Y, Zhang J, Chen X (2021) Lymphocyte-to-Monocyte Ratio is Associated with the Poor Prognosis of Breast Cancer Patients Receiving Neoadjuvant Chemotherapy. Cancer Manag Res Volume 13:1571-1580. https://doi.org/10.2147/CMAR.S292048

17. Patel DA, Xi J, Luo J et al (2019) Neutrophil-to-lymphocyte ratio as a predictor of survival in patients with triple-negative breast cancer. Breast Cancer Res Treat 174:443-452. https://doi.org/10.1007/s10549-018-05106-7

18. Ivars Rubio A, Yufera JC, de la Morena P et al (2019) Neutrophil-lymphocyte ratio in metastatic breast cancer is not an independent predictor of survival, but depends on other variables. Sci Rep 9:16979. https://doi.org/10.1038/s41598-019-53606-3

19. Ni X-J, Zhang X-L, Ou-Yang Q-W et al (2014) An Elevated Peripheral Blood Lymphocyte-to-Monocyte Ratio Predicts Favorable Response and Prognosis in Locally Advanced Breast Cancer following Neoadjuvant Chemotherapy. PLoS ONE 9:e111886. https://doi.org/10.1371/journal.pone.0111886

20. Hayes DF, Ethier S, Lippman ME (2006) New guidelines for reporting of tumor marker studies in breast cancer research and treatment: REMARK. Breast Cancer Res Treat 100:237-238. https://doi.org/10.1007/s10549-006-9253-5

21. Schwartz LH, Seymour L, Litière S et al (2016) RECIST 1.1 - Standardisation and disease-specific adaptations: Perspectives from the RECIST Working Group. Eur J Cancer 62:138-145. https://doi.org/10.1016/j.ejca.2016.03.082

22. Wei B, Yao M, Xing C et al (2016) The neutrophil lymphocyte ratio is associated with breast cancer prognosis: an updated systematic review and meta-analysis. OncoTargets Ther Volume 9:55675575. https://doi.org/10.2147/OTT.S108419

23. Liu X, Qu J-K, Zhang J et al (2017) Prognostic role of pretreatment neutrophil to lymphocyte ratio in breast cancer patients: A meta-analysis. Med (Baltim) 96:e8101. https://doi.org/10.1097/MD.0000000000008101

24. Noh H, Eomm M, Han A (2013) Usefulness of Pretreatment Neutrophil to Lymphocyte Ratio in Predicting Disease-Specific Survival in Breast Cancer Patients. J Breast Cancer 16:55. https://doi.org/10.4048/jbc.2013.16.1.55

25. Wariss BR, de Souza Abrahão K, de Aguiar SS et al (2017) Effectiveness of four inflammatory markers in predicting prognosis in 2374 women with breast cancer. Maturitas 101:51-56. 
https://doi.org/10.1016/j.maturitas.2017.04.015

26. de la Cruz-Ku G, Chambergo-Michilot D, Torres-Roman JS et al (2020) Neutrophil-to-lymphocyte ratio predicts early mortality in females with metastatic triple-negative breast cancer. PLoS ONE 15:e0243447. https://doi.org/10.1371/journal.pone.0243447

27. Sato H, Tsubosa Y, Kawano T (2012) Correlation Between the Pretherapeutic Neutrophil to Lymphocyte Ratio and the Pathologic Response to Neoadjuvant Chemotherapy in Patients With Advanced Esophageal Cancer. World J Surg 36:617-622. https://doi.org/10.1007/s00268-011-14111

28. Ferrando-Martínez S, Franco JM, Hernandez A et al (2009) Thymopoiesis in elderly human is associated with systemic inflammatory status. AGE 31:87-97. https://doi.org/10.1007/s11357-0089084-x

29. Manuel M, Tredan O, Bachelot T et al (2012) Lymphopenia combined with low TCR diversity (divpenia) predicts poor overall survival in metastatic breast cancer patients. Oncolmmunology 1:432-440. https://doi.org/10.4161/onci.19545

30. Schettini F, Sobhani N, lanza A et al (2020) Immune system and angiogenesis-related potential surrogate biomarkers of response to everolimus-based treatment in hormone receptor-positive breast cancer: an exploratory study. Breast Cancer Res Treat 184:421-431. https://doi.org/10.1007/s10549-020-05856-3

31. Emile G, Penager S, Levy C et al (2021) Baseline lymphopenia as prognostic factor in patients with metastatic breast cancer treated with palbociclib. Oncol Lett 23:25. https://doi.org/10.3892/ol.2021.13143

32. Mahmoud SMA, Paish EC, Powe DG et al (2011) Tumor-Infiltrating CD8 ${ }^{+}$Lymphocytes Predict Clinical Outcome in Breast Cancer. J Clin Oncol 29:1949-1955. https://doi.org/10.1200/JC0.2010.30.5037

33. Coffelt SB, Kersten K, Doornebal CW et al (2015) IL-17-producing $\gamma \delta T$ cells and neutrophils conspire to promote breast cancer metastasis. Nature 522:345-348. https://doi.org/10.1038/nature14282

34. Goel S, DeCristo MJ, Watt AC et al (2017) CDK4/6 inhibition triggers anti-tumour immunity. Nature 548:471-475. https://doi.org/10.1038/nature23465

35. Deng J, Wang ES, Jenkins RW et al (2018) CDK4/6 Inhibition Augments Antitumor Immunity by Enhancing T-cell Activation. Cancer Discov 8:216-233. https://doi.org/10.1158/2159-8290.CD-170915

36. Templeton AJ, Ace O, McNamara MG et al (2014) Prognostic Role of Platelet to Lymphocyte Ratio in Solid Tumors: A Systematic Review and Meta-Analysis. Cancer Epidemiol Biomarkers Prev 23:12041212. https://doi.org/10.1158/1055-9965.EPI-14-0146

37. Hu Y, Wang S, Ding N et al (2020) Platelet/Lymphocyte Ratio Is Superior to Neutrophil/Lymphocyte Ratio as a Predictor of Chemotherapy Response and Disease-free Survival in Luminal B-like (HER2-) Breast Cancer. Clin Breast Cancer 20:e403-e409. https://doi.org/10.1016/j.clbc.2020.01.008 
38. Cho U, Park HS, Im SY et al (2018) Prognostic value of systemic inflammatory markers and development of a nomogram in breast cancer. PLoS ONE 13:e0200936. https://doi.org/10.1371/journal.pone.0200936

39. Krenn-Pilko S, Langsenlehner U, Thurner E-M et al (2014) The elevated preoperative platelet-tolymphocyte ratio predicts poor prognosis in breast cancer patients. Br J Cancer 110:2524-2530. https://doi.org/10.1038/bjc.2014.163

40. Suzuki K, Aiura K, Ueda M, Kitajima M (2004) The Influence of Platelets on the Promotion of Invasion by Tumor Cells and Inhibition by Antiplatelet Agents. Pancreas 29:132-140. https://doi.org/10.1097/00006676-200408000-00008

41. Klinger MHF, Jelkmann W (2002) Review: Role of Blood Platelets in Infection and Inflammation. J Interferon Cytokine Res 22:913-922. https://doi.org/10.1089/10799900260286623

42. Nishijima TF, Muss HB, Shachar SS et al (2015) Prognostic value of lymphocyte-to-monocyte ratio in patients with solid tumors: A systematic review and meta-analysis. Cancer Treat Rev 41:971-978. https://doi.org/10.1016/j.ctrv.2015.10.003

43. Ma Y, Zhang J, Chen X (2021) Lymphocyte-to-Monocyte Ratio is Associated with the Poor Prognosis of Breast Cancer Patients Receiving Neoadjuvant Chemotherapy. Cancer Manag Res Volume 13:1571-1580. https://doi.org/10.2147/CMAR.S292048

44. Cesaire M, Le Mauff B, Rambeau A et al (2020) Mécanismes de la lymphopénie radio-induite et implications thérapeutiques. Bull Cancer (Paris) 107:813-822. https://doi.org/10.1016/j.bulcan.2020.04.009

45. Kumar R, Geuna E, Michalarea V et al (2015) The neutrophil-lymphocyte ratio and its utilisation for the management of cancer patients in early clinical trials. Br J Cancer 112:1157-1165. https://doi.org/10.1038/bjc.2015.67

\section{Figures}




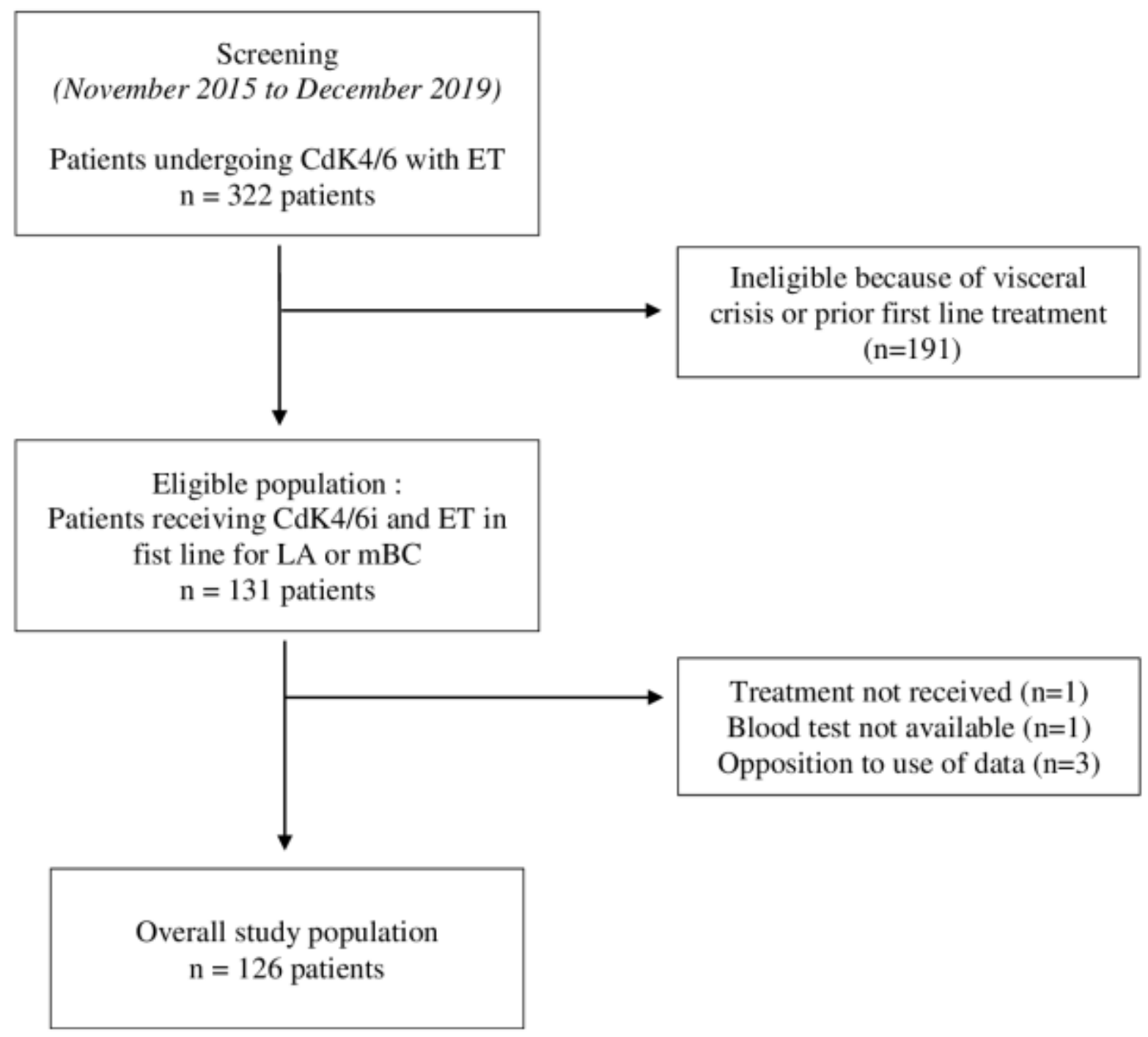

Figure 1

Flow-chart 


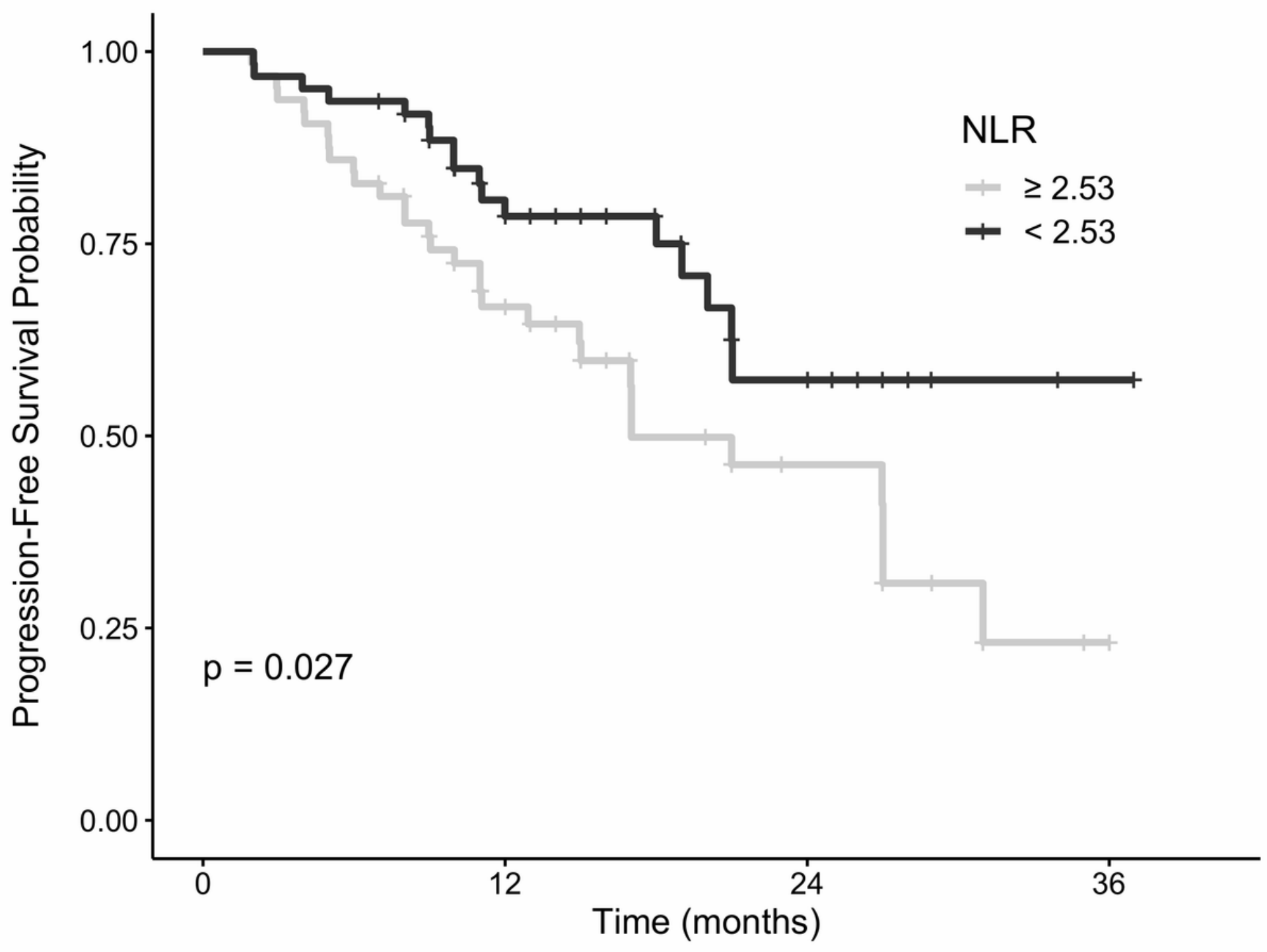

Number at risk

\begin{tabular}{|c|c|c|c|c|}
\hline$\frac{\underline{\alpha}}{\vec{\Sigma}^{2}}<2.53$. & $\begin{array}{l}64 \\
62\end{array}$ & $\begin{array}{l}33 \\
37\end{array}$ & $\begin{array}{c}9 \\
11\end{array}$ & 1 \\
\hline & 0 & 12 & & 36 \\
\hline
\end{tabular}

Figure 2

PFS probability according to pretreatment NLR 

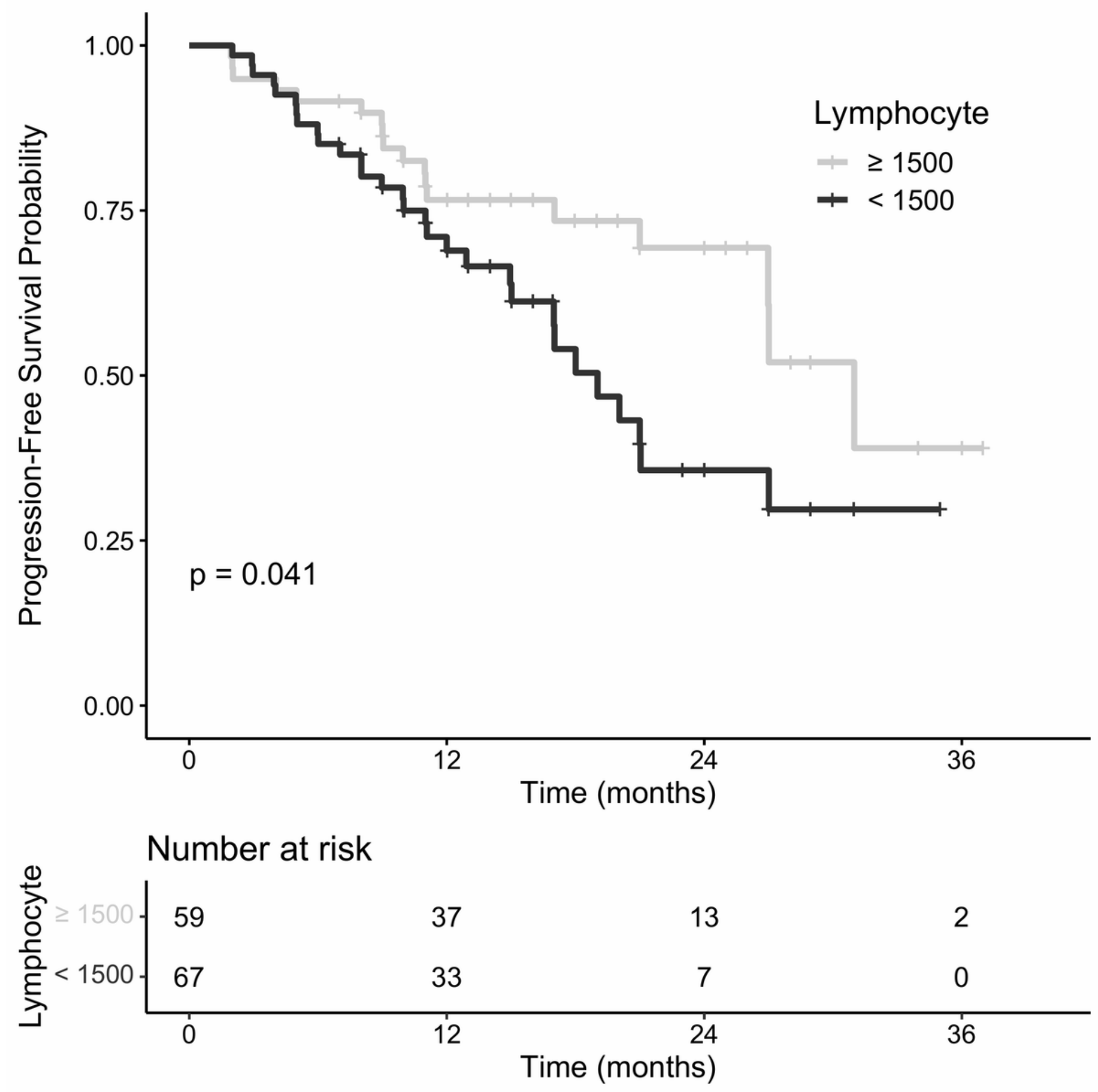

Figure 3

PFS probability according to pretreatment ALC 